\title{
SUPPLEMENTARY NOTE ON THE FREQUENCIES OF COMPLEMENTARY HUES
}

\author{
BY \\ IRWIN G. PRIEST
}

Referring to the "Note on the Relation Between the Frequencies of Complementary Hues," 1 Dr. Selig Hecht has kindly pointed out in a letter to the author, that, if the relation between frequencies is hyperbolic, it follows of mathematical necessity that the relation between wave-lengths is also hyperbolic. This is true. It might therefore be thought a matter of indifference as to whether frequency or wave-length is considered in presenting such data. There is, however, another aspect of the question: If frequency scales be used, the experimental data fall symmetrically about the vertex of the hyperbola. (J. Op. Soc. Am., 52 p...404, Fig. 2). If wave-length scales be used, two-thirds of the same data fall on one side of the vertex. (J. Op. Soc. Am., h, p. 404, Fig. 1.)

It appears therefore that, as regards the treatment of experimental data, the relation can be more conveniently and accurately demonstrated on frequency scales. These are the points worthy of notice. The author regrets that in emphasizing them he was led to imply the contradiction of a mathematical theorem.

It may be noted, that the asymptotes of these hyperbolic equations correspond roughly to the "neutral points" in the spectra of the "red-green blind" and the "yellow-blue blind" respectively, thus:

In vibrations per trillionth of one second,

Asymptotes of Hyperbola

530

608
Neutral Points

522 yellow-blue blind (not well known)

612-601 red-green blind. 
In words, the spectral region lying between the "neutral points" for these two general classes of "color blinds" is approximately coextensive with the region for which the normal eye finds no spectral complementary.

BUREAU OF STANDARDS

Nov. 5, 1921. 